\title{
BMJ Open Induced pluripotent stem cell-based Drug Repurposing for Amyotrophic lateral sclerosis Medicine (iDReAM) study: protocol for a phase I dose escalation study of bosutinib for amyotrophic lateral sclerosis patients
}

Keiko Imamura, ${ }^{1}$ Yuishin Izumi, ${ }^{2}$ Haruhiko Banno, ${ }^{1}$ Ryuji Uozumi (D) , ${ }^{3}$ Satoshi Morita, ${ }^{3}$ Naohiro Egawa, ${ }^{4}$ Takashi Ayaki, ${ }^{4}$ Makiko Nagai, ${ }^{5}$ Kazutoshi Nishiyama, ${ }^{5}$ Yasuhiro Watanabe, ${ }^{6}$ Ritsuko Hanajima, ${ }^{6}$ Ryosuke Oki, ${ }^{2}$ Koji Fujita, ${ }^{2}$ Naoto Takahashi, ${ }^{7}$ Takafumi Ikeda, ${ }^{8}$ Akira Shimizu, ${ }^{8}$ Akiko Morinaga, ${ }^{9}$ Tomoko Hirohashi, ${ }^{9}$ Yosuke Fujii, ${ }^{9}$ Ryosuke Takahashi, ${ }^{4}$ Haruhisa Inoue (i) ${ }^{1}$

To cite: Imamura K, Izumi Y, Banno $\mathrm{H}$, et al. Induced pluripotent stem cell-based Drug Repurposing for Amyotrophic lateral sclerosis Medicine (iDReAM) study: protocol for a phase I dose escalation study of bosutinib for amyotrophic lateral sclerosis patients. BMJ Open 2019;9:e033131. doi:10.1136/ bmjopen-2019-033131

- Prepublication history for this paper is available online. To view these files, please visit the journal online (http://dx.doi org/10.1136/bmjopen-2019033131).

Received 22 July 2019 Revised 23 October 2019 Accepted 07 November 2019

Check for updates

(C) Author(s) (or their employer(s)) 2019. Re-use permitted under CC BY-NC. No commercial re-use. See rights and permissions. Published by BMJ.

For numbered affiliations see end of article.

Correspondence to Professor Haruhisa Inoue; haruhisa@cira.kyoto-u.ac.jp

\section{ABSTRACT}

Introduction Amyotrophic lateral sclerosis (ALS) is a progressive and severe neurodegenerative disease caused by motor neuron death. There have as yet been no fundamental curative medicines, and the development of a medicine for ALS is urgently required. Induced pluripotent stem cell (iPSC)-based drug repurposing identified an Src/c-Abl inhibitor, bosutinib, as a candidate molecular targeted therapy for ALS. The objectives of this study are to evaluate the safety and tolerability of bosutinib for the treatment of patients with ALS and to explore the efficacy of bosutinib on ALS. This study is the first clinical trial of administered bosutinib for patients with ALS.

Methods and analysis An open-label, multicentre phase I dose escalation study has been designed. The study consists of a 12-week observation period, a 1-week transitional period, a 12-week study treatment period and a 4-week follow-up period. After completion of the transitional period, subjects whose total ALS Functional Rating Scale-Revised (ALSFRS-R) score decreased by 1-3 points during the 12-week observation period receive bosutinib for 12 weeks. Three to six patients with ALS are enrolled in each of the four bosutinib dose levels $(100,200,300$ or $400 \mathrm{mg} /$ day) to evaluate the safety and tolerability under a $3+3$ dose escalation study design. Dose escalation and maximum tolerated dose are determined by the safety assessment committee comprising oncologists/ haematologists and neurologists based on the incidence of dose-limiting toxicity in the first 4 weeks of the treatment at each dose level. A recommended phase II dose is determined by the safety assessment committee on completion of the 12-week study treatment in all subjects at all dose levels. The efficacy of bosutinib is also evaluated exploratorily using ALS clinical scores and biomarkers.

Ethics and dissemination This study received full ethical approval from the institutional review board of each participating site. The findings of the study will be

\section{Strengths and limitations of this study}

This is the first study to use an Src/c-Abl inhibitor, bosutinib, for patients with amyotrophic lateral sclerosis (ALS), and is considered to be the first step in the development of a molecular-targeted therapy for ALS.

- This study presents a novel type of protocol design by combining the safety study of oncology and the efficacy study of neurology.

- Limitations are the open-label and single-arm designs for safety and tolerability.

disseminated in peer-reviewed journals and at scientific conferences.

Trial registration number UMIN000036295; Pre-results, JMA-IIA00419; Pre-results.

\section{INTRODUCTION}

Amyotrophic lateral sclerosis (ALS) is an intractable neurodegenerative disease caused by the death of motor neurons resulting in progressive skeletal muscle weakness. The worldwide incidence of ALS is approximately 2 per 100000 persons per year. ${ }^{1}$ About $10 \%$ of patients with ALS have familial inheritance, and in Japan, ${ }^{2}$ around $30 \%$ of those patients have SOD1 gene mutation. ${ }^{3}$ Although the disease mechanism of ALS is still unknown, motor neuron death and accumulation of misfolded proteins are essential pathological characteristics of the disease. In the late stage of the disease, patients lose their spontaneous motor function and present 
respiratory failure. The survival period is within $3-5$ years after onset if a mechanical ventilator is not adopted. ${ }^{4}$ To date, riluzole ${ }^{5}$ and edaravone ${ }^{6}$ have been approved for ALS treatment, although there are no fundamental curative medicines.

We previously identified bosutinib, an Src/c-Abl inhibitor, as resulting in an increase in the survival rate of ALS motor neurons derived from familial ALS with SOD1 mutation and from sporadic ALS patients' induced pluripotent stem cells (iPSCs). Bosutinib improved the impaired autophagy, reduced the accumulation of misfolded proteins and attenuated the energy shortage of ALS patient motor neurons. ${ }^{7}$ Furthermore, treatment with bosutinib attenuated the ALS-related phenotypes of ALS model mice. ${ }^{78}$ Penetration of the blood-brain barrier was confirmed by a previous report. ${ }^{9}$ Based on these findings, we hypothesised that bosutinib, as a molecular targeted therapy, would attenuate the progression of muscle weakness and elongate the survival period of patients with ALS according to its pathomechanism-dependent effects, and thus we designed the clinical trial of bosutinib for patients with ALS.

Bosutinib is a selective inhibitor of Src/c-Abl tyrosine kinase, approved for the treatment of chronic myelogenous leukaemia (CML). In September 2012, the US Food and Drug Administration (FDA) approved bosutinib for the treatment of CML, chronic, accelerated or blast phase Philadelphia chromosome-positive CML, for those who are resistant to or who cannot tolerate other therapies including imatinib. Then, FDA granted accelerated approval of bosutinib for the treatment of patients with newly diagnosed CML in December 2017. Although known frequent adverse effects include diarrhoea, thrombocytopenia and liver transaminase elevations, ${ }^{10}$ from the results of past clinical trials with patients with CML, it became clear that the safety of bosutinib can be managed. However, because the disease-related physical conditions of patients with ALS are different from those of patients with CML, evaluation of the safety and tolerability of bosutinib in patients with ALS was planned to be conducted in the present study. Also, evaluation of the efficacy of bosutinib in patients with ALS using ALS Functional Rating Scale-Revised (ALSFRS-R) ${ }^{11}$ and biomarkers was designed to be conducted in an exploratory manner.

\section{METHODS}

\section{Study design}

This study is an investigator-initiated, open-label, multicentre, phase I dose escalation study to evaluate the safety and tolerability of bosutinib for determination of the maximum tolerated dose (MTD) and a recommended phase II dose (RP2D) in patients with ALS. Efficacy is also evaluated exploratorily.

The following patients will be included in this study.

- Patients with sporadic ALS diagnosed with isolated definite, probable or probable-laboratory supported ALS as determined by the Updated Awaji Criteria, ${ }^{12}$ or patients diagnosed with ALS with progressive muscle weakness and a superoxide dismutase 1 (SOD1) mutation (which has already been reported).

- Patients with Grade 1 or 2 ALS according to ALS severity classification as determined by the Japanese Ministry of Health, Labour and Welfare (MHLW) Specific Disease Research Group in Japan (box 1); patients with Grade 3 ALS with a SOD1 mutation are allowed to be enrolled.

- Patients with ALS within 2 years after onset at the time of primary enrolment (patients with ALS with an SOD1 mutation within 5 years after the onset are allowed to be enrolled).

- Patients with total ALSFRS-R score reduced by 1-3 points during the observation period.

Detailed eligibility criteria are presented in box 2. In order to avoid undesirable carry-over effects caused by previous medications, patients taking edaravone were excluded and riluzole use during the study treatment period was prohibited. To ensure patient safety and to evaluate the safety of bosutinib appropriately, bulbar type ALS was excluded.

The study consists of a 12-week observation period, a 1-week transitional period, a 12-week study treatment period and a 4-week follow-up period (figure 1). Subjects who have been receiving riluzole are allowed to continue to take riluzole during the 12-week observation period (with the dosage remaining unchanged), but they must stop taking riluzole from the beginning of the 1-week transitional period. After completion of the transitional period, subjects whose total ALSFRS-R score decreased by 1-3 points during the 12-week observation period will receive bosutinib for 12 weeks to evaluate its safety and tolerability in patients with ALS. All ALS drugs including riluzole will be prohibited during the bosutinib treatment period. In this study, three to six patients with ALS will be enrolled at each of the four bosutinib dose levels

\section{Box 1 Criteria for severity of amyotrophic lateral} sclerosis (ALS) (Ministry of Health and Welfare Study Group on Neurodegenerative Diseases as Specified Diseases, 1998)

- Grade 1: Able to work or perform housework; movement disturbance of one limb or dysarthria caused by bulbar palsy.

- Grade 2: Independent living but inconveniences in daily life; movement disorders of one or two limbs, trunk, tongue, face, palate, larynx.

- Grade 3: Requiring assistance for eating, excretion or ambulation; three or more muscle weaknesses in limbs, trunk, tongue, face, palate and larynx.

- Grade 4: Need assistance in everyday life; presence of respiratory insufficiency, difficulty in coughing out sputum or dysphagia.

- Grade 5: Bedridden and full life support equipment required.

(Source: FDA edaravone medical review/MCl186-19-Protocol, Section 16.1.1, p250/267)

ALSFRS-R, ALS Functional Rating Scale-Revised. 


\section{Box 2 Eligibility criteria}

\section{Inclusion criteria:}

1. Evidence of a personally signed and dated informed consent document indicating that the patient has been informed of all pertinent aspects of the study. To be additionally signed by a delegate signer if the subject is unable to handwrite.

2. Patients aged $\geq 20$ years and $<80$ years at the time of informed consent.

3. Patients with positive already-reported SOD1 gene mutation and progressive muscle weakness; patients with sporadic ALS who are categorised as either 'Definite ALS' or 'Probable ALS' or 'Probablelaboratory supported ALS' in the Updated Awaji Criteria for the diagnosis of ALS.

4. Patients at Grade 1 or 2 in the Japan ALS Severity Scale of the grant-in-aid programme for chronic diseases from the Japanese Ministry of Health, Labour and Welfare; patients with positive SOD1 mutation of Grade 1, 2 or 3.

5. Patients with ALS that occurred within 2 years at the time of the first registration; patients with positive SOD1 mutation within 5 years after disease onset

6. Patients who can visit hospitals regularly as outpatients.

7. Patients with change in total ALSFRS-R score during the observation period from -1 to -3 points.

8. Urine pregnancy test (for female of childbearing potential) negative at screening.

Female patients of non-childbearing potential must meet at least one of the following criteria:

a. Achieved postmenopausal status defined as follows: cessation of regular menses for at least 12 consecutive months with no alternative pathological or physiological cause; status may be confirmed with a serum follicle-stimulating hormone level confirming the postmenopausal state.

b. Have undergone a documented hysterectomy and/or bilateral oophorectomy.

c. Have medically confirmed ovarian failure.

All other female patients (including female patients with tubal ligations) are considered to be of childbearing potential.

Male and female patients of childbearing potential must agree to use one highly effective method of contraception as outlined in this protocol, throughout the study and for at least 28 days after the last dose of investigational product.

9. Patients with appropriate renal function as defined as follows at the time of the first and second registrations.

a. Serum creatinine $\leq 1.5 \times$ upper limit of normal (ULN) or estimated creatinine clearance $\geq 60 \mathrm{~mL} / \mathrm{min}$ as calculated using the standard method for the institution.

10. Patients with appropriate hepatic function as defined as follows at the time of the first and second registrations.

a. Total serum bilirubin $\leq 1.5 \times$ ULN unless the patient has documented Gilbert syndrome.

b. AST and ALT $\leq 2.5 \times$ ULN.

11. Able to take tablets orally.

12. Patients whose acute effect of previous treatment has recovered to the baseline or CTCAE v.4.03 $\leq$ Grade 1 at the time of the first and second registrations.

13. Willing and able to comply with scheduled visits, treatment plan, laboratory tests and other study procedures.

\section{Exclusion criteria:}

1. Patients with tracheostomy.
Box 2 Continued

2. Patients who have used non-invasive ventilation due to ALS symptoms.

3. Patients whose $\%$ Forced vital capacity (FVC) is less than $70 \%$ at the time of first and second registrations.

4. Patients who have nerve conduction study findings of demyelination such as conduction block.

5. Patients who are taking edaravone; patients who started riluzole or edaravone after start of the observation period; patients who changed the dosage of riluzole after start of the observation period.

6. Patients with bulbar type ALS with dysphagia and dysarthria.

7. Patients with cognitive impairment.

8. Pregnant female patients; breastfeeding female patients; fertile male and female patients of childbearing potential who are unwilling or unable to use one highly effective method of contraception as outlined in this protocol for the duration of the study and for at least 28 days after the last dose of the investigational product.

9. History of clinically significant or uncontrolled cardiac disease including:

- History of, or active, congestive heart failure.

- Uncontrolled angina or hypertension within 3 months prior to registration.

- Myocardial infarction within 12 months prior to registration.

- Clinically significant ventricular arrhythmia (such as ventricular tachycardia, ventricular fibrillation or Torsades de pointes).

- Diagnosed or suspected congenital or acquired prolonged QT interval history or prolonged QTc (QTcF should not exceed $500 \mathrm{~ms}$ ).

- Unexplained syncope.

10. Uncontrolled hypomagnesaemia or uncorrected hypokalaemia due to potential effects on the QT interval.

11. Patient who is taking the following medicines during study drugs administration.

a. Combination of warfarin anticoagulation or related oral anticoagulation. Combination of therapeutic anticoagulant therapy with low molecular weight heparin is acceptable.

b. Src or c-Abl inhibitors.

c. Other treatments for cancer.

d. Drugs known to prolong the QT interval or predispose to Torsades de Pointes.

e. Current or anticipated use of a strong or moderate CYP3A inhibitor and inducer.

f. Drugs affecting gastric pH such as proton pump inhibitors (eg, lansoprazole).

12. History of malignancy within 5 years prior to registration with the exception of basal cell carcinoma or cervical carcinoma in situ or stage 1 or 2 cancer that is considered adequately treated and currently in complete remission for at least 12 months.

13. Patients who were enrolled in other clinical study within 12 weeks before the first registration or are expected to be enrolled in other clinical study using a study drug during this study.

14. Known prior or suspected severe hypersensitivity to study drugs or any component in their formulations.

15. Patients with active, uncontrolled bacterial, fungal or viral infection, including hepatitis B virus, hepatitis C virus, known HIV or AIDSrelated illness.

16. Recent or ongoing clinically significant GI disorder (eg, Crohn's disease, ulcerative colitis, or prior total or partial gastrectomy).

17. Patients with chronic obstructive pulmonary disease.

Continued

Continued 


\section{Box 2 Continued}

18. Major surgery or radiotherapy within 14 days prior to registration at the time of the first registration.

19. Patient who fulfils the conditions:

a. Neutrophil count $\left(\right.$ ANC) $<1500 / \mathrm{mm}^{3}$ or white cell count $<3000 /$ $\mathrm{mm}^{3}$ at the time of the first and second registrations.

b. Haemoglobin $<9.0 \mathrm{~g} / \mathrm{dL}$ at the time of the first and second registrations.

c. Platelet count $<100000 / \mathrm{L}$ at the time of the first and second registrations.

20. Other acute or chronic medical or psychiatric condition including recent (within the past year) or active suicidal ideation or behaviour or laboratory abnormality that may increase the risk associated with study participation or investigational product administration or may interfere with the interpretation of study results and, in the judgement of the investigator, would make the participant inappropriate for entry into this study.

21. Investigation site staff members directly involved in the conduct of the study and their family members, site staff members otherwise supervised by the investigator (subinvestigator), including their family members.

ALS, amyotrophic lateral sclerosis; ALSFRS-R, ALS Functional Rating ScaleRevised;

$\mathrm{Gl}$, gastrointestinal.

(100 mg/day (dose level 1), $200 \mathrm{mg}$ /day (dose level 2), $300 \mathrm{mg} /$ day (dose level 3) or $400 \mathrm{mg} /$ day (dose level 4) ) to evaluate the safety and tolerability of the investigational drug (bosutinib) under a 3+3 dose escalation study design $^{13}$ (figure 2). The dose will be escalated by one dose level at a time; no skipping will be allowed. Dose escalation and MTD will be determined based on assessment by the safety assessment committee consisting of oncologists and haematologists, as well as a neurological expert who is also an ALS expert, as measured by the incidence of dose-limiting toxicity (DLT) for 4 weeks after initiating each dose level. The definition of DLT is shown in box 3. RP2D is the dosage selected according to the results of a phase I study for uses in future studies

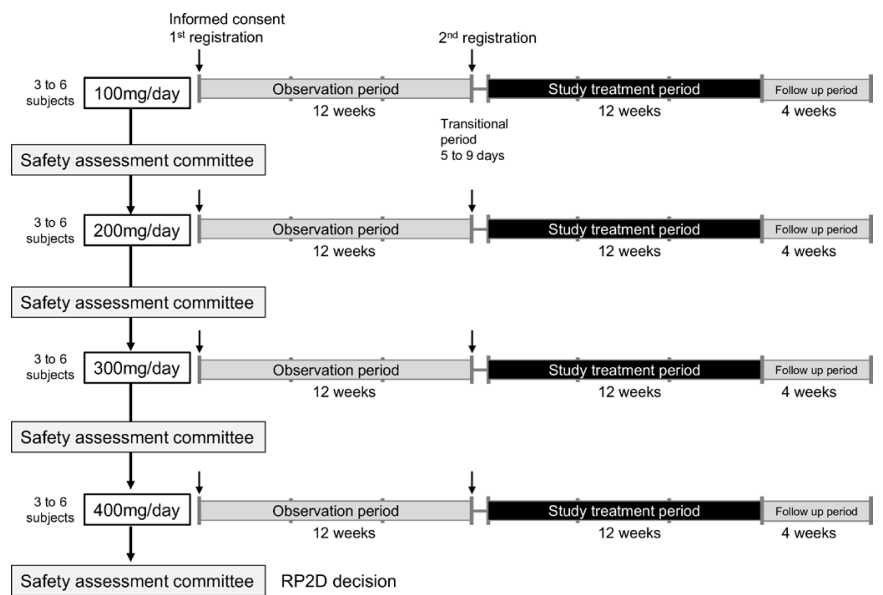

Figure 1 Study schedule of the iDReAM (Induced pluripotent stem cell-based Drug Repurposing for Amyotrophic lateral sclerosis Medicine) study.

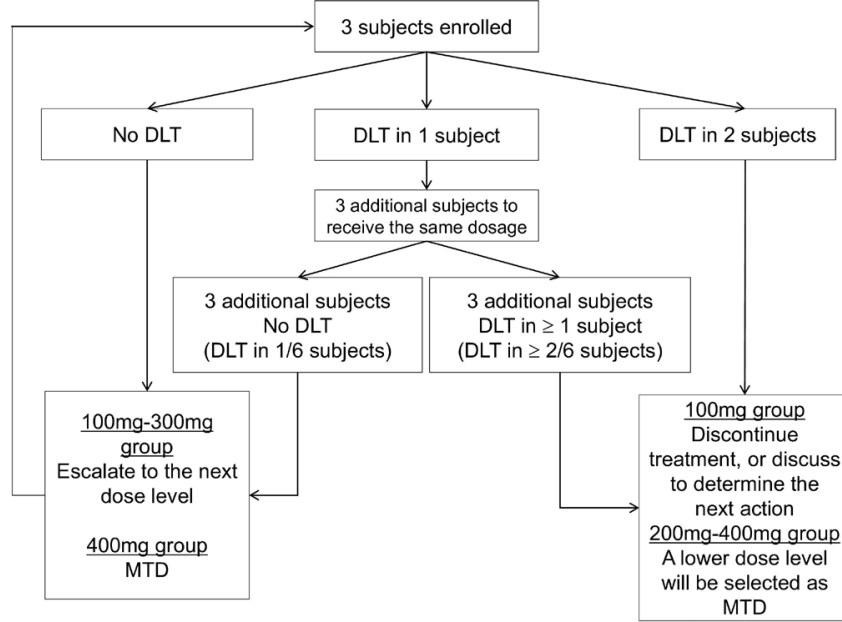

Figure $23+3$ dose escalation study design in the iDReAM (Induced pluripotent stem cell-based Drug Repurposing for Amyotrophic lateral sclerosis Medicine) study. DLT, doselimiting toxicity.

and will be projected as a recommended clinical dose for patients with ALS. Thus, in order to confirm its suitability for long-term clinical administration, RP2D will be selected comprehensively based on the evaluation of all safety data obtained (including DLT, dose intensity and other relevant data throughout the treatment period), as well as pharmacological data, exploratory endpoints and other relevant data.

The target sample size of this clinical trial is 12-24 subjects. The number of subjects at each dose level of this study will depend on the results of the DLT assessments.

\section{Box 3 Definition of DLT}

- Grade 4 neutropenia $\left(<500 / \mathrm{mm}^{3}\right)$ persisting for $>7$ day duration or Grade 4 white cell count decrease for $>7$ day duration $\left(<1000 / \mathrm{mm}^{3}\right)$.

- Febrile neutropenia (ANC $<1000 / \mathrm{mm}^{3}$ and fever $\geq 38.5^{\circ} \mathrm{C}$ ).

- Grade 4 thrombocytopenia $>48$ hour duration or with bleeding requiring platelet transfusion.

- Any Grade 3 or 4 clinically evident non-haematological toxicity - except for $\geq$ Grade 3 nausea, vomiting or diarrhoea unless subject is on optimal medical therapy, or

- electrolyte abnormality requiring hospitalisation, or is life-threatening.

$\geq 66 \%$ of bosutinib doses that cannot be completed due to an adverse event related to the investigational drug (during the first 4 weeks of treatment and the entire 12 weeks of treatment).

- An event otherwise determined as a DLT by principal (sub) investigator and the safety assessment committee.

- Any clinically significant $\geq$ Grade 2 toxicity that requires $>14$ days to resolve (to $\leq$ Grade 1 despite optimal medical therapy).

Remarks: Respiratory failure is a very important clinical event for amyotrophic lateral sclerosis (ALS). Thus, dyspnoea, hypoxia, pneumonitis, pulmonary oedema and pulmonary fibrosis according to CTCAE V.4.03 will be considered as 'clinically relevant $\geq$ Grade 2 adverse events.

Toxicity of bosutinib in patients with ALS is not known.Dose-limiting toxicity (DLT) criteria may be modified or added if it is considered necessary to include additional DLT criteria deemed appropriate for patients with ALS based on information accumulated from this study. 


\section{Outcomes}

The primary endpoint is DLT during the first 4 weeks of treatment with bosutinib, and during the study treatment period (12 weeks). Adverse events, abnormal laboratory test results, vital signs (blood pressure, pulse rate, body temperature), ECG and chest X-ray findings are evaluated as secondary endpoints. Adverse events are graded based on the National Cancer Institute (NCI) Common Terminology Criteria for Adverse Events (CTCAE) V.4.03. Pharmacokinetic assessments will be conducted for subjects who have serious adverse events. Changes in ALS clinical score including total ALSFRS-R score and in the grade of the Japan ALS severity classification, and changes from the baseline of \% FVC and grip power are evaluated as exploratory endpoints. Changes in blood neurofilament L (NF-L) and phosphorylated neurofilament $\mathrm{H}$ (pNF-H) during the observation period and the study treatment period are also evaluated as exploratory endpoints.

\section{Data analysis and statistical method}

The frequency of DLT during the study treatment period will be analysed for each dose level. The frequencies of adverse events during the study treatment period are analysed for each dose group. Onset timing, severity and the relationship of the study drug to adverse events are summarised. The respective correlations of changes in total ALSFRS-R score versus blood NF-L and blood pNF-H will be assessed.

\section{Patient and public involvement}

Patients and the public were not involved in the design of this study.

\section{Registration}

This study has been planning since March 2019 to March 2021, and participating four sites in Japan.

\section{DISCUSSION}

This study evaluates the safety and tolerability of bosutinib for the molecularly targeted treatment of patients with ALS as well as explores the efficacy of bosutinib on ALS. The potential efficacy of bosutinib for patients with ALS has already been identified by an approach applying iPSC-based drug repurposing. ${ }^{7}$ We previously developed a phenotypic screen system to evaluate compounds with a readout of motor neuron survival using ALS patientiPSCs with SOD1 mutations. ${ }^{7}$ Application of the screen revealed that bosutinib increased the survival rate of ALS motor neurons. This drug was also effective for sporadic ALS patient-iPSC models. Thus, we designed the clinical trial for ALS patients with SOD1 mutation and for patients with sporadic ALS, with the expectation that bosutinib will become a candidate molecularly targeted drug for ALS.

Bosutinib is a therapeutic agent used for the treatment of patients with CML, and the accumulated data of previous clinical trials presenting the safety of bosutinib can also be managed in patients with CML. However, evaluation of the safety and tolerability of bosutinib in patients with ALS is required in order to support the development of bosutinib for the treatment of ALS. This clinical trial was designed as a 'triple 3 design' by a combination of the safety study, the $3+3$ dose escalation study design of oncology and the efficacy study, with a 3-month observation period for uniform subject inclusion, of neurology.

Safety information has been accumulated from clinical studies of bosutinib in patients with GML. Known frequent adverse effects of bosutinib include diarrhoea $(70 \%)$, thrombocytopenia (35\%) and liver enzyme abnormalities (alanine aminotransferase increase of $31 \%$, aspartate aminotransferase increase of 23\%) according to the Bosutinib Trial in First-Line Chronic Myelogenous Leukemia Treatment (BFORE) trial, which demonstrated the efficacy of bosutinib as first-line treatment in adult patients with newly diagnosed chronic-phase CML. ${ }^{10}$ While diarrhoea is the most commonly reported adverse event, it is generally manageable by dietary advice to avoid dairy products, high fat content and spicy food before anti-diarrhoeal agents. ${ }^{10}$ Patients with haematological adverse events or liver transaminase elevations are managed by dose interruption or reduction. ${ }^{10}$ The occurrence and management of adverse events in patients with ALS seem to be comparable to those with CML. While referring to the accumulated clinical data of CML, this study was designed to evaluate any unacceptable adverse events that specifically occur in patients with ALS, as well as the treatment tolerability in patients with ALS. Given that a 4-week DLT evaluation period was included in the phase I/II study to determine MTD in patients with CML (NCT00811070), a 4-week DLT evaluation period is included in this study to determine MTD with bosutinib in patients with ALS. In addition to MTD, this study determines RP2D considered optimal for patients with ALS. After discussion with the regulatory authority referencing the clinical data of CML, 12 weeks of the study treatment period is considered necessary to determine RP2D in the current study. Regarding the dose, a $400 \mathrm{mg}$ dose of bosutinib has been approved by FDA as a first-line treatment for CML. For the non-oncology study of bosutinib, a phase 2 study has been conducted in patients with autosomal dominant polycystic kidney disease, in which bosutinib at starting doses of 200 and $400 \mathrm{mg} /$ day was administered. ${ }^{14}$ Thus, up to $400 \mathrm{mg}$ of bosutinib dose was selected for evaluation of the safety and tolerability for patients with ALS in this study. Regarding the drug interaction, CYP3A inhibitors/inducers are prohibited because bosutinib is primarily metabolised by CYP3A. Drugs affecting gastric $\mathrm{pH}$ are also prohibited because blood concentration of bosutinib may be decreased, resulting in reduced efficacy.

Patients with ALS eventually develop degeneration of both upper and lower motor neurons, but their symptoms and disease processes vary. ${ }^{15}$ In the early stage of the disease, only upper or lower motor neuron signs may manifest themselves. Some patients also continue to exhibit either one of the motor neuron signs for relatively 
long periods of time. Especially, ALS patients with an SOD1 mutation often do not meet typical ALS diagnostic criteria because they lack upper motor neuron signs, and they sometimes present a different clinical prognosis from sporadic ALS, ${ }^{16}$ showing slow progression in some cases. Thus, inclusion criteria contained special provisions for ALS patients with an SOD1 mutation.

The variety of clinical symptoms and disease progression speed among patients is one of the reasons that cause difficulty for clinical trial evaluation. In the current study, an observation period of 12 weeks was established to enable the evaluation of ALS patients with uniform progression speed, the same as in previous clinical trials with edaravone, which was approved for ALS to cause a delay in motor function decline. ${ }^{6}$ To explore the efficacy of bosutinib, a change in ALS clinical score such as the ALSFRS-R score, the grade in Japan ALS severity classification, a change from baseline of \% FVC and a change in grip power are all evaluated as exploratory endpoints. In addition, blood NF-L levels and pNF-H levels, which have been reported to be increased in blood and cerebrospinal fluid of patients with ALS, ${ }^{17-19}$ are also evaluated as an indicator of the effectiveness of bosutinib. These biomarkers might indicate the degree of motor neuron death, and its alteration might reflect the effect of bosutinib treatment.

This study is the first to use an Src/c-Abl inhibitor, bosutinib, for patients with ALS, and it presents a novel type of protocol design by combining a safety study for oncology and an efficacy study for neurology. This is a phase I study for the safety and tolerability evaluation, and the number of subjects was limited. Therefore, it will be necessary to conduct further safety and efficacy evaluations in the following phases. This study is considered to be the first step in the development of a molecularly targeted drug for ALS therapy.

\section{Ethics and dissemination}

This study was approved by the Institutional Review Boards of Kyoto University, Tokushima University, Kitasato University and Tottori University. This study was registered with the University Hospital Medical Information Network clinical trials registry (UMIN) Clinical Trials Registry (UMIN000036295) and the Japan Medical Association Center for Clinical Trials (JMACCT CTR) (JMA-IIA00419).

\footnotetext{
Author affiliations

${ }^{1}$ Center for iPS Cell Research and Application (CiRA), Kyoto University, Kyoto, Japan ${ }^{2}$ Department of Neurology, Tokushima University Graduate School of Biomedical Sciences, Tokushima, Japan

${ }^{3}$ Department of Biomedical Statistics and Bioinformatics, Kyoto University, Kyoto, Japan

${ }^{4}$ Department of Neurology, Graduate School of Medicine, Kyoto University, Kyoto, Japan

${ }^{5}$ Department of Neurology, Kitasato University School of Medicine, Sagamihara, Japan

${ }^{6}$ Division of Neurology, Department of Brain and Neurosciences, Faculty of Medicine, Tottori University, Yonago, Japan
}

${ }^{7}$ Department of Hematology, Nephrology, and Rheumatology, Akita University Graduate School of Medicine, Akita, , Japan

${ }^{8}$ Institute for Advancement of Clinical and Translational Science (iACT), Kyoto University Hospital, Kyoto, Japan

${ }^{9}$ Pfizer R\&D Japan G.K, Tokyo, Japan

\section{Twitter Takafumi lkeda @takafumios}

Acknowledgements We appreciate the advice on the protocol from Manabu Muto, Toshiyuki Kitano, Hirofumi Maruyama, Satoshi Kaneko, and Mitsumasa Watanabe, and the protocol review and study preparation from Pfizer Japan and its global medicine team of bosutinib. We would like to express our sincere gratitude to all our coworkers and collaborators, to Atsushi Onodera and Satoshi Nishihama for making the contracts, to Nozomi Kawabata, Mikie lijima and Azusa Okayama for their administrative support, and to Peter Karagiannis for critical reading.

Contributors $\mathrm{KI}, \mathrm{AM}, \mathrm{TH}, \mathrm{YF}$ and $\mathrm{HI}$ made contributions to the conception and design of the study protocol. RU and SM provided statistical expertise. $\mathrm{YI}, \mathrm{HB}, \mathrm{NE}$, TA, MN, KN, YW, RH, RO, KF, NT, TI, AS and RT made clinical suggestions for the design of the protocol.

Funding This research was funded in part by a grant for Clinical Research and Trials for the Clinical Innovation Network (CIN) and Realization of Regenerative Medicine from the Japan Agency for Medical Research and Development (AMED) (19lk0201083h0002), and the Research Project for Practical Applications of Regenerative Medicine (18bk0104019h0001) from AMED to HI.

Competing interests The study drug bosutinib and the pharmacokinetic analysis for only subject-experienced serious adverse events are supported by Pfizer JAPAN under the Clinical Research Collaboration Agreement. RT and NT received research funding and honoraria for lectures from Pfizer.

Patient consent for publication Not required.

Provenance and peer review Not commissioned; externally peer reviewed.

Data availability statement Data are available in a public, open access repository.

Open access This is an open access article distributed in accordance with the Creative Commons Attribution Non Commercial (CC BY-NC 4.0) license, which permits others to distribute, remix, adapt, build upon this work non-commercially, and license their derivative works on different terms, provided the original work is properly cited, appropriate credit is given, any changes made indicated, and the use is non-commercial. See: http://creativecommons.org/licenses/by-nc/4.0/.

\section{ORCID iDs}

Ryuji Uozumi http://orcid.org/0000-0002-9546-9869

Haruhisa Inoue http://orcid.org/0000-0003-4736-9537

\section{REFERENCES}

1 Marin B, Boumédiene F, Logroscino G, et al. Variation in worldwide incidence of amyotrophic lateral sclerosis: a meta-analysis. Int J Epidemiol 2017;46:57-74.

2 Nakamura R, Sone J, Atsuta N, et al. Next-Generation sequencing of 28 ALS-related genes in a Japanese ALS cohort. Neurobiol Aging 2016;39:219.e1-219.e8.

3 Finsterer J, Burgunder J-M. Recent progress in the genetics of motor neuron disease. Eur J Med Genet 2014:57:103-12.

4 Maurel C, Dangoumau A, Marouillat S, et al. Causative genes in amyotrophic lateral sclerosis and protein degradation pathways: a link to neurodegeneration. Mol Neurobiol 2018;55:6480-99.

5 Bensimon G, Lacomblez L, Meininger V, et al. A controlled trial of riluzole in amyotrophic lateral sclerosis. ALS/Riluzole Study Group. $N$ Engl J Med 1994;330:585-91.

6 Abe K, Aoki M, Tsuji S, et al. Safety and efficacy of edaravone in well defined patients with amyotrophic lateral sclerosis: a randomised, double-blind, placebo-controlled trial. The Lancet Neurology 2017;16:505-12.

7 Imamura K, Izumi Y, Watanabe A, et al. The Src/c-Abl pathway is a potential therapeutic target in amyotrophic lateral sclerosis. Sci Trans/ Med 2017;9. doi:10.1126/scitransImed.aaf3962

8 Wenqiang C, Lonskaya I, Hebron ML, et al. Parkin-Mediated reduction of nuclear and soluble TDP-43 reverses behavioral decline in symptomatic mice. Hum Mol Genet 2014;23:4960-9.

9 Lonskaya I, Hebron ML, Desforges NM, et al. Tyrosine kinase inhibition increases functional parkin-Beclin-1 interaction and enhances amyloid clearance and cognitive performance. EMBO Mol Med 2013;5:1247-62. 
10 Cortes JE, Apperley JF, DeAngelo DJ, et al. Management of adverse events associated with bosutinib treatment of chronic-phase chronic myeloid leukemia: expert panel review. J Hematol Oncol 2018;11.

11 Cedarbaum JM, Stambler N, Malta E, et al. The ALSFRS-R: a revised ALS functional rating scale that incorporates assessments of respiratory function. BDNF ALS Study Group (phase III). J Neurol Sci 1999;169:13-21.

12 Costa J, Swash M, de Carvalho M, et al. Awaji criteria for the diagnosis of amyotrophic lateral sclerosis:a systematic review. Arch Neurol 2012;69:1410-6.

13 Jaki T, Clive S, Weir CJ. Principles of dose finding studies in cancer: a comparison of trial designs. Cancer Chemother Pharmacol 2013;71:1107-14.

14 Tesar V, Ciechanowski K, Pei Y, et al. Bosutinib versus placebo for autosomal dominant polycystic kidney disease. J Am Soc Nephrol 2017;28:3404-13.
15 Nishiyama A, Niihori T, Warita $\mathrm{H}$, et al. Comprehensive targeted next-generation sequencing in Japanese familial amyotrophic lateral sclerosis. Neurobiol Aging 2017;53:194.e1-194.e8.

16 Bali T, Self W, Liu J, et al. Defining SOD1 ALS natural history to guide therapeutic clinical trial design. J Neurol Neurosurg Psychiatry 2017;88:99-105.

17 De Schaepdryver M, Jeromin A, Gille B, et al. Comparison of elevated phosphorylated neurofilament heavy chains in serum and cerebrospinal fluid of patients with amyotrophic lateral sclerosis. $J$ Neurol Neurosurg Psychiatry 2018;89:367-73.

18 Verde F, Steinacker P, Weishaupt JH, et al. Neurofilament light chain in serum for the diagnosis of amyotrophic lateral sclerosis. J Neurol Neurosurg Psychiatry 2019;90:157-64.

19 Poesen K, Van Damme P. Diagnostic and prognostic performance of neurofilaments in ALS. Front Neurol 2018;9:1167. 